\title{
Fully endoscopic microvascular decompression for hemifacial spasm
}

\author{
Tracy M. Flanders, MD, ${ }^{1}$ Rachel Blue, MD, ${ }^{1}$ Sanford Roberts, MD, ${ }^{1}$ Brendan J. McShane, BA, ${ }^{1}$ \\ Bryan Wilent, PhD, DABNM, ${ }^{2}$ Vijay Tambi, MS, DABNM, ${ }^{2}$ Dmitriy Petrov, MD, ${ }^{1}$ and \\ John Y. K. Lee, MD, MSCE'
}

${ }^{1}$ Department of Neurosurgery, University of Pennsylvania, Philadelphia, Pennsylvania; and ${ }^{2}$ SpecialtyCare, Nashville, Tennessee

\begin{abstract}
OBJECTIVE Hemifacial spasm (HFS) is characterized by involuntary tonic and/or clonic contractions of facial nerve muscles. Fully endoscopic microvascular decompression (E-MVD) for HFS has not been widely adopted. This paper aims to illustrate the safety and efficacy of the fully endoscopic technique for HFS treatment.

METHODS The authors conducted a single-center retrospective study of 27 patients (28 separate E-MVD cases; 1 patient had bilateral E-MVD) diagnosed with HFS who underwent fully E-MVD from January 2013 to October 2016. Intraoperative brainstem auditory evoked potentials and lateral spread resolution were reviewed. Outcome was based on the clinical status of the patient at the last contact point with the senior author. Complications were categorized as facial weakness, hearing loss, ataxia, dysphagia, or any adverse event able to be attributed to the surgical procedure.

RESULTS HFS was relieved either completely or partially in the majority of cases ( 24 of $28,85.7 \%$ ). Of the 28 separate procedures, $17(60.7 \%)$ resulted in complete resolution of symptoms, $4(14.3 \%)$ resulted in near-complete resolution, 2 $(7.1 \%)$ resulted in 50\% reduction of symptoms, 1 (3.6\%) resulted in minimal reduction, and $4(14.3 \%)$ resulted in no relief. Of the 27 patients, 26 (96\%) had no permanent postoperative complications. In multivariate logistic regression, the best predictor of greater than $50 \%$ resolution of spasm was resolution of intraoperative lateral spread response.
\end{abstract}

CONCLUSIONS A fully E-MVD for HFS provides a safe and comprehensive view of the neurovascular conflict. Exclusive use of the endoscope in MVD is both safe and feasible in the treatment of HFS. Attention to lateral spread response monitoring remains an integral part of comprehensive neurosurgical management.

https://thejns.org/doi/abs/10.3171/2018.4.JNS172631

KEYWORDS hemifacial spasm; endoscopic; microvascular decompression; lateral spread response; functional neurosurgery

$\mathrm{H}$ EMIFACIAL spasm (HFS) is the unilateral tonic and/ or clonic contraction of facial muscles. Beginning in the orbicularis oculi muscle with subsequent eyelid closure and/or eyebrow elevation, the symptoms often progress to the frontalis, platysma, and orbicularis oris muscles. ${ }^{39}$ In its most severe form, HFS can present as a disfiguring, sustained grimace of partial eye closure and mouth lifting, described as the "tonus phenomenon." ${ }^{34}$ The involuntary facial movements have various psychosocial consequences for patients and can be highly debilitating. Patients often report symptom exacerbation with fatigue, anxiety, and/or head movement. ${ }^{2}$
Focal compression of the facial nerve resulting in myelin breakdown and ephaptic transmission is the accepted pathophysiology for HFS. ${ }^{26}$ The root exit zone is recognized as the myelination transition of oligodendrocyte to Schwann cells; this region of the facial nerve is thus only protected by the arachnoid mater and is highly vulnerable to vascular insults. ${ }^{30}$ The anterior inferior cerebellar artery (AICA), posterior inferior cerebellar artery (PICA), and vertebral artery (VA) are the most frequently implicated vessels in HFS. ${ }^{31}$

In 1962, Gardner first described the treatment of HFS with elimination of the neurovascular conflict between

ABBREVIATIONS AICA = anterior inferior cerebellar artery; BAEP = brainstem auditory evoked potential; $\mathrm{CPA}=$ cerebellopontine angle; $\mathrm{EA}-\mathrm{MVD}=\mathrm{endoscope-assisted}$ MVD; E-MVD = endoscopic MVD; HFS = hemifacial spasm; IONM = intraoperative neuromonitoring; LSR = lateral spread response; MVD = microvascular decompression; $\mathrm{PICA}=$ posterior inferior cerebellar artery; $\mathrm{VA}=$ vertebral artery.

SUBMITTED October 19, 2017. ACCEPTED April 17, 2018.

INCLUDE WHEN CITING Published online October 5, 2018; DOI: 10.3171/2018.4.JNS172631. 
compressive arterial vessels and the facial nerve. ${ }^{8}$ Jannetta et al. revolutionized the treatment of HFS with microvascular decompression (MVD) via the suboccipital approach to the cerebellopontine angle (CPA). MVD has now become the standard and accepted surgical technique for HFS treatment with long-term success rates (within 10 years) of $83 \%-97 \% .5,14$

In recent years, endoscopy has entered the neurosurgical arena as an attractive alternative or adjunct for conventional microsurgery. ${ }^{1,715-17,20,27,28,33}$ The endoscope provides the advantage over the microscope of enhanced and safe visualization of the neurovascular anatomy. ${ }^{23}$ The panoramic view afforded by the endoscope enlarges the surgical field of vision while eliminating cerebellar or brainstem retraction and the need for extensive dissection, which is necessary when using the microscope, to allow for an unobstructed view of the relevant neurovascular structures. Additionally, the use of angled lenses allows for visualization around corners, which would otherwise be visually obstructed. In cadaveric studies, quantitative analysis has demonstrated a nearly 2-fold increase in the view of the endoscope compared with the microscope. ${ }^{35}$ This enhanced visualization has, in our experience and in commentary of other surgeons, allowed for a greater anatomical exposure that enables an increased ability to locate nerve-vessel conflicts that are limited with the microscope vantage point. ${ }^{4}$ While the endoscope has been adopted by neurosurgeons as an adjunct to microsurgery in the posterior fossa, there are limited data on fully endoscopic microvascular decompression (E-MVD) for HFS. The current study aimed to present one surgeon's case series of HFS patients undergoing fully E-MVD to illustrate the safety and efficacy of the fully endoscopic technique.

\section{Methods \\ Study Participants}

The University of Pennsylvania institutional review board approved this study, and a waiver of HIPAA Authorization was obtained as data were retrospectively analyzed. Between January 2013 and October 2016, 27 patients with a preoperative diagnosis of HFS underwent fully E-MVD by the senior author (J.Y.K.L.) in the Department of Neurosurgery at Pennsylvania Hospital. Patients were selected for elective treatment of their HFS based on clinical history and physical examination and radiological findings. Imaging studies were provided to note the presence of structural abnormalities and neurovascular conflicts. All patients underwent clinical evaluation preoperatively and postoperatively by the senior author or another healthcare provider at the same institution. The results in the present study represent only those patients who chose to undergo surgical treatment for HFS. Patients who underwent microscope only or combined microscope and endoscope-assisted MVD (EA-MVD) were excluded.

\section{Operative Procedure}

The surgeon performed 28 fully E-MVDs between 2013 and 2016. Prior to 2013, the surgeon had performed microscopic surgery exclusively and microscopic surgery with endoscopic assistance (EA-MVD), in which the mi- croscope was used as the primary visualization tool and the endoscope as an adjunct. In the present study, the surgeon employed a fully endoscopic technique. Further details of the operative technique and early experiences with MVD for trigeminal neuralgia have been extensively described in prior publications. , $^{3,11,22,23}$ In particular, the position of the scope relative to the surgeon's hands has been extensively described as well. ${ }^{23}$ In this fully endoscopic approach, the Mitaka Pneumatic Arm (Mitake Kohki Co.) was utilized with a $2.7-\mathrm{mm}, 0^{\circ}$ endoscope (Storz). The small diameter of the endoscope allows for maximization of the surgical working space to allow for entrée of other instruments (Fig. 1). The $30^{\circ}$-angled endoscope is employed to enhance further visualization of the CPA.

\section{Intraoperative Neuromonitoring}

The intraoperative neuromonitoring (IONM) plan for each patient included free-run and triggered cranial nerve electromyography, brainstem auditory evoked potentials (BAEPs), and lateral spread response (LSR) monitoring. IONM was performed using a Cascade (Cadwell) or NIM-Eclipse (Medtronic) system. Electromyography activity was recorded from needle electrodes inserted intradermally in the frontalis, orbicularis oculi, orbicularis

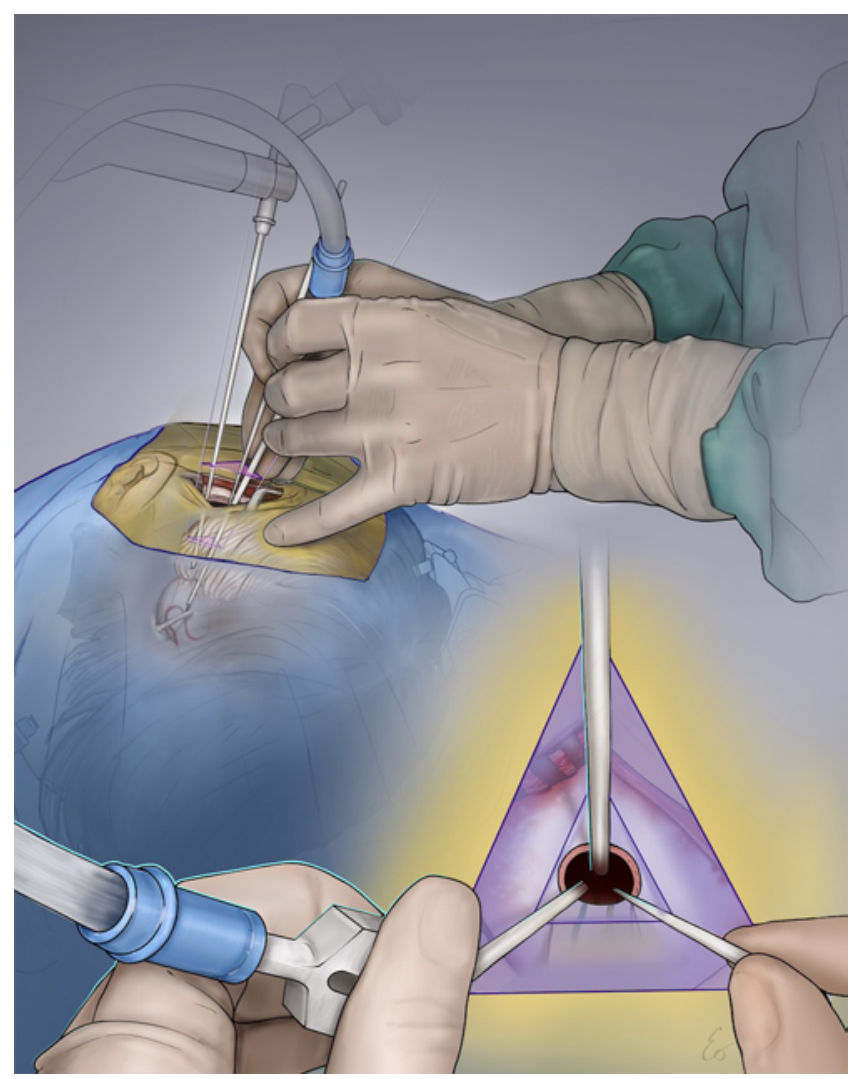

FIG. 1. Illustration of the operative technique employed by the senior author for the fully endoscopic approach. The position of the surgeon's hands in relation to the endoscope with the Mitaka Pneumatic Arm has been well described in prior publications. ${ }^{23}$ Artist Eo Trueblood. Copyright Stream Studios at the Children's Hospital of Philadelphia. Published with permission. Figure is available in color online only. 
oris, and mentalis muscles. LSR was evoked by stimulation of the zygomatic branch of the ipsilateral facial nerve from 6 to $12 \mathrm{~mA}$. LSRs were recorded from the ipsilateral orbicularis oris and mentalis muscles. Short-acting depolarizing muscle relaxants were administered at induction and then maintained at minimal doses to preserve signals from the ulnar nerve, the median nerve, and the posterior tibial nerve. For BAEPs, alternating rarefaction and condensation clicks were delivered individually to each ear at 85 - to $95-\mathrm{dB}-\mathrm{SPL}$ intensity at a rate of $11.1-17.5 \mathrm{~Hz}$. While one ear was being stimulated, contralateral white noise masking 40-65 dB-SPL was applied. BAEPs were recorded via subdermal needles placed at A1, A2, and $\mathrm{CPz}$ with channels of $\mathrm{A} 1-\mathrm{CPz}$ and $\mathrm{A} 2-\mathrm{CPz}$ for the left and right ears, respectively. All BAEPs were bandpass filtered from 100 to $1000 \mathrm{~Hz}$, and 200-500 trials were used for $\mathrm{BAEP}$ recording.

\section{Clinical Follow-Up Review}

Outcome was based on the clinical status of the patient at the last contact point with the senior author or with a clinician in the hospital system. The shortest follow-up time was less than 1 month. Outcome was determined by the effect of treatment on signs and symptoms. Complications were categorized as facial weakness, hearing loss, ataxia, dysphagia, or any adverse event that could be attributed to the surgical procedure.

\section{Statistical Analysis}

All patients were grouped into one of 2 groups based on clinical outcome: 1) resolution of symptoms or 2) presence of residual symptoms. Patients were also grouped based on intraoperative lateral spread findings: 1) complete resolution or 2) no resolution. Two-by-two contingency tables were created, and Fisher's exact test was used to compare groups due to low numbers, with $\alpha$ set at 0.05 . Stata 10 (StataCorp) was used for analysis of contingency tables as well as of univariate and multivariate logistic regression.

\section{Results \\ Patient Demographics}

Patient demographics are summarized in Table 1. There were 9 men (33.3\%) and 18 women (66.7\%). The mean age was 54.6 years (range 28-73 years). Of the 27 patients, $26(96.3 \%)$ had previously undergone failed treatment with Botox. There were 16 left-sided (57.1\%) and 12 right-sided $(42.9 \%)$ cases; of note, 1 patient had bilateral HFS and underwent staged (4 months later) bilateral EMVD. Of the 27 patients, 2 patients $(7.4 \%$ ) had undergone prior ipsilateral MVD for HFS: one patient underwent 3 total ipsilateral MVDs prior to surgical treatment by the senior author (2 to treat HFS and 1 to treat trigeminal neuralgia), and the other patient underwent ipsilateral MVD by the senior author 2 years prior for HFS before implementation of the fully E-MVD. In addition, 1 patient had a stable, calcified meningioma posterior to the hypoglossal canal and above the foramen magnum in addition to HFS, and she underwent biopsy of this lesion during E-MVD. The mean duration of symptoms prior to surgical treatment was 6 years (range 1.5-17 years).
TABLE 1. Patient demographics

\begin{tabular}{lc}
\hline \multicolumn{3}{l}{} & Value \\
\hline Sex & $9(33.3)$ \\
\hline Male & $18(66.7)$ \\
\hline Female & $54.6(28-73)$ \\
\hline Mean age, yrs (range) & $26(96.3)$ \\
\hline Preop Botox & $1(3.7)$ \\
\hline Yes & $16(57.1)$ \\
\hline No & $12(42.9)$ \\
\hline Laterality & $2(7.4)$ \\
\hline Lt & $25(92.6)$ \\
\hline Rt & $6.0(1.5-17)$ \\
\hline Prior ipsilateral HFS op & \\
\hline Yes & \\
\hline No & \\
\hline Mean duration of Sx prior to op, yrs (range) &
\end{tabular}

\section{Intraoperative Findings: Operating Room Data and Offending Vessel}

Intraoperative findings are summarized in Table 2. The mean operating room time, defined as from the start of skin incision to the end of skin closure, was 119.7 minutes with a range of 87 to 206 minutes.

Of the 28 E-MVDs, 19 (67.9\%) were notable for BAEP changes, and $9(32.1 \%)$ did not exhibit any BAEP changes. Of the 19 with BAEP changes, 5 (26.3\%) returned to baseline, and $14(73.7 \%)$ returned to near baseline at du-

TABLE 2. Intraoperative findings

\begin{tabular}{lc}
\hline \multicolumn{2}{l}{ Value } \\
\hline Mean op room time, mins (range) & $119.7(87-206)$ \\
\hline BAEP changes & $19(67.9)$ \\
\hline Yes & $9(32.1)$ \\
\hline No & $5(26.3)$ \\
\hline BAEP return to baseline prior to dural closure \\
\hline Yes & $14(73.7)$ \\
\hline No & $16(57.1)$ \\
\hline Resolution of LSR w/ decompression & $10(35.7)$ \\
\hline Yes & $2(7.1)$ \\
\hline No & $19(67.9)$ \\
\hline Unknown & $2(7.1)$ \\
\hline Vessel & $3(10.7)$ \\
\hline AICA & $4(14.3)$ \\
\hline AlCA \& other vessel & \\
\hline Other* & \\
\hline Unknown &
\end{tabular}

J Neurosurg Volume $131 \cdot$ September 2019 
ral closure. With regard to lateral spread, 16 (57.1\%) cases exhibited complete resolution with decompression of the facial nerve and $10(35.7 \%)$ did not have resolution of the LSR; 2 patients $(7.1 \%$ ) had unknown responses (one patient had no lateral spread that could be evoked at the time of surgery, and the other patient's IONM data were unable to be located).

The neurovascular conflict was attributed to the following vessels: 19 (67.9\%) AICAs, 2 AICAs and another vessel (7.1\%) (AICA and VA; and AICA and brainstem perforator), $3(10.7 \%)$ other (transverse pontine vein or small arteriole; VA; and VA and PICA). The vessels were unknown in $4(14.3 \%)$ cases (operative notes did not explicitly identify the offending vessel).

\section{Postoperative Data and Complications}

Postoperative information and complications are summarized in Table 3. The mean length of stay was 3 days (range 2-7 days). The mean follow-up with the senior author was 2.9 months (range 0.25-27 months, mode 1 month). Of the 27 patients, 26 (96\%) patients had no permanent complications, and 20 (74\%) had no postoperative complications at all. Only 1 patient had an objective permanent neurological complication, hearing loss, which was confirmed by specialist referral. This patient did not have BAEP changes at the time of surgery. One patient (3.7\%) exhibited postoperative ataxia but refused vestibular rehabilitation or further treatment, and the symptoms resolved over time. One patient (3.7\%) reported subjective dysphagia but did not require feeding tube placement or further workup, and the symptoms resolved over time. Three patients (11.1\%) had transient facial weakness that resolved at the final clinic visit, and all 3 of these patients had previously been treated with Botox injections. One patient (3.7\%) had significant transient pain from dried blood thought to be from an IONM needle resulting in otitis that resolved over time. CSF leak, cardiac events, stroke, and mortality did not occur in any of the patients postoperatively.

Clinical outcomes are summarized in Table 3. Of the 28 separate E-MVD cases ( 27 patients, 1 with bilateral E-MVD), 17 (60.7\%) experienced complete resolution of symptoms, $4(14.3 \%)$ had near-complete resolution, 2 (7.1\%) had 50\% reduction of symptoms, $1(3.6 \%)$ had minimal reduction, and 4 (14.3\%) had no relief.

\section{IONM Findings and Correlation to Postoperative Outcomes}

As summarized in Table 2, of the 28 E-MVDs, 19 $(67.9 \%)$ had a $>1.0$ msec shift in wave V of the BAEP, while $9(32.1 \%)$ did not. Of the 19 cases, 5 (26.3\%) returned to baseline, and 14 (73.7\%) returned to near baseline at dural closure. Of the 19 cases with BAEP changes that had complete or near-complete return to baseline, none of the patients exhibited postoperative hearing loss. For the 9 cases that did not have $>1.0$-msec shift in wave $\mathrm{V}, 1$ patient did exhibit postoperative hearing loss, and this patient was noted to have a $0.7-\mathrm{msec}$ shift in wave $\mathrm{V}$ on retrospective review. LSR data were available in 26 of 28 cases. Sixteen patients (61.5\%) had complete resolution
TABLE 3. Postoperative findings

\begin{tabular}{lc}
\hline & Value \\
\hline Mean LOS, days (range) & $3.0(2-7)$ \\
\hline Follow-up w/ senior author, mos & $2.9(0.25-27)$ \\
\hline Mean (range) & 1 \\
\hline Mode & $1(3.7)$ \\
\hline Permanent complications & 0 \\
\hline Partial hearing loss & 0 \\
\hline Cardiac event & 0 \\
\hline Stroke & $3(11.1)$ \\
\hline Death & 0 \\
\hline Subjective/transient complications & $1(3.7)$ \\
\hline Transient facial palsy & $1(3.7)$ \\
\hline Permanent facial palsy & $1(3.7)$ \\
\hline Ataxia & 0 \\
\hline Dysphagia & \\
\hline Otitis & $17(60.7)$ \\
\hline CSF leak & $4(14.3)$ \\
\hline Resolution of Sx & $2(7.1)$ \\
\hline Complete resolution & $1(3.6)$ \\
\hline Near-complete & $4(14.3)$ \\
\hline $50 \%$ reduction &
\end{tabular}

LOS = length of stay.

Values are presented as the number of patients (\%) unless stated otherwise.

* Values are presented as the number of cases (\%).

with decompression of the facial nerve, and 10 (38.4\%) did not. For one patient, an LSR could not be obtained at baseline, and for another, the IONM record was not retrievable.

As summarized in Tables 4 and 5, intraoperative resolution of lateral spread resulted in a 7-fold higher chance of achieving more than 50\% spasm-free relief $(p=0.036)$. The following variables were tested in a stepwise logistic regression in multivariate analysis: sex, age (years), prior Botox injection, prior ipsilateral MVD, and duration of symptoms (years). In patients who experienced $\geq 50 \%$ spasm relief, resolution of LSR was found to have an OR of $26.59(\mathrm{p}=0.020)$.

\section{Discussion}

Since the late 1960s, MVD has been viewed as the standard of care for HFS treatment when medical manage-

TABLE 4. Prediction of spasm-free outcome with resolution of lateral spread

\begin{tabular}{lcc}
\hline & $\begin{array}{c}\text { Univariate Analysis } \\
\text { p Value }\end{array}$ & $\begin{array}{c}\text { Logistic Regression } \\
\text { Analysis OR }\end{array}$ \\
\hline $100 \%$ spasm relief & 0.15 & 3.3 \\
\hline$\geq 90 \%$ spasm relief & 0.074 & 4.5 \\
\hline$\geq 50 \%$ spasm relief & 0.036 & 7 \\
\hline
\end{tabular}


TABLE 5. Multivariate analysis of spasm-free outcome

\begin{tabular}{ccc}
\hline & $\begin{array}{c}\text { Multivariate } \\
\text { Analysis p Value }\end{array}$ & $\begin{array}{c}\text { Logistic Regression } \\
\text { Analysis OR }\end{array}$ \\
\hline $100 \%$ spasm relief & 0.103 & 4.58 \\
\hline $290 \%$ spasm relief & 0.05 & 6 \\
\hline$\geq 50 \%$ spasm relief & 0.02 & 26.59 \\
\hline
\end{tabular}

ment has failed. ${ }^{14,30}$ We propose that fully E-MVD confers superior visualization of the complex anatomy of the CPA. While many studies have examined fully endoscopic decompression of the trigeminal nerve, there are few reports of fully E-MVD for HFS. Cheng et al. reported 10 cases of fully E-MVD for HFS and stated that all of the patients experienced full resolution of symptoms postoperatively. ${ }^{4}$ They concluded that fully endoscopic approaches allow for identification of neurovascular conflicts that may not be clearly seen via the microscope.

Several studies have proven that IONM with BAEPs significantly reduces the risk of postoperative hearing loss. ${ }^{9,18,25,32}$ In the present study, 1 patient experienced ipsilateral postoperative hearing loss. This is comparable to reports of postoperative hearing deficits found in other studies. ${ }^{29,32}$ However, IONM during this particular patient's E-MVD in our series did not reveal a significant change in BAEPs during the decompression (i.e., greater than 1.0-msec shift in wave V). On retrospective review, the patient was noted to have a $0.7-\mathrm{msec}$ shift in wave V, which suggests that more conservative alert criteria, such as $0.5 \mathrm{msec}$, may be warranted. The goal of IONM is near zero false negatives while limiting the false positives. The alert criteria of $1 \mathrm{msec}$ is set to balance sensitivity and specificity. ${ }^{36}$ In retrospect, a more prudent criterion may be a 0.5 -msec shift from baseline set prior to dura opening. The patient's shift of $0.7 \mathrm{msec}$ is more in line with these stricter criteria. Furthermore, the drilling, fluid deposition, or bone dust in the middle ear associated with a craniectomy can also produce hearing loss that may evolve in the postoperative period. ${ }^{43}$ Further investigation of BAEPs in the context of fully endoscopic EVD is necessary to elucidate this mismatch between intraoperative BAEPs and postoperative hearing loss and, in this case, the cause of hearing loss is still not entirely clear.

LSR serves as an intraoperative surrogate to adequate facial nerve decompression. ${ }^{10,13,42}$ The phenomenon viewed on IONM presumes that resolution of lateral spread correlates with adequate decompression of the facial nerve (Fig. 2). However, the use of LSR as a prognostic indicator of HFS outcome is relatively controversial. Some studies have found that intraoperative LSR does not correlate with postdecompression outcome in HFS. , 24,38 Other studies have reported that intraoperative LSR accurately predicts clinical outcome. ${ }^{19}$ A meta-analysis showed that if the LSR is abolished intraoperatively, the success rate of achieving symptom relief will be $90.5 \%$ but will drop to $61 \%$ when the LSR cannot be abolished intraoperatively. ${ }^{34}$ A study of 322 HFS patients showed a significant predictive value of LSR and a success rate of $94.7 \%$ when the LSR was abolished and only $67.3 \%$ when it is not. ${ }^{37}$ The

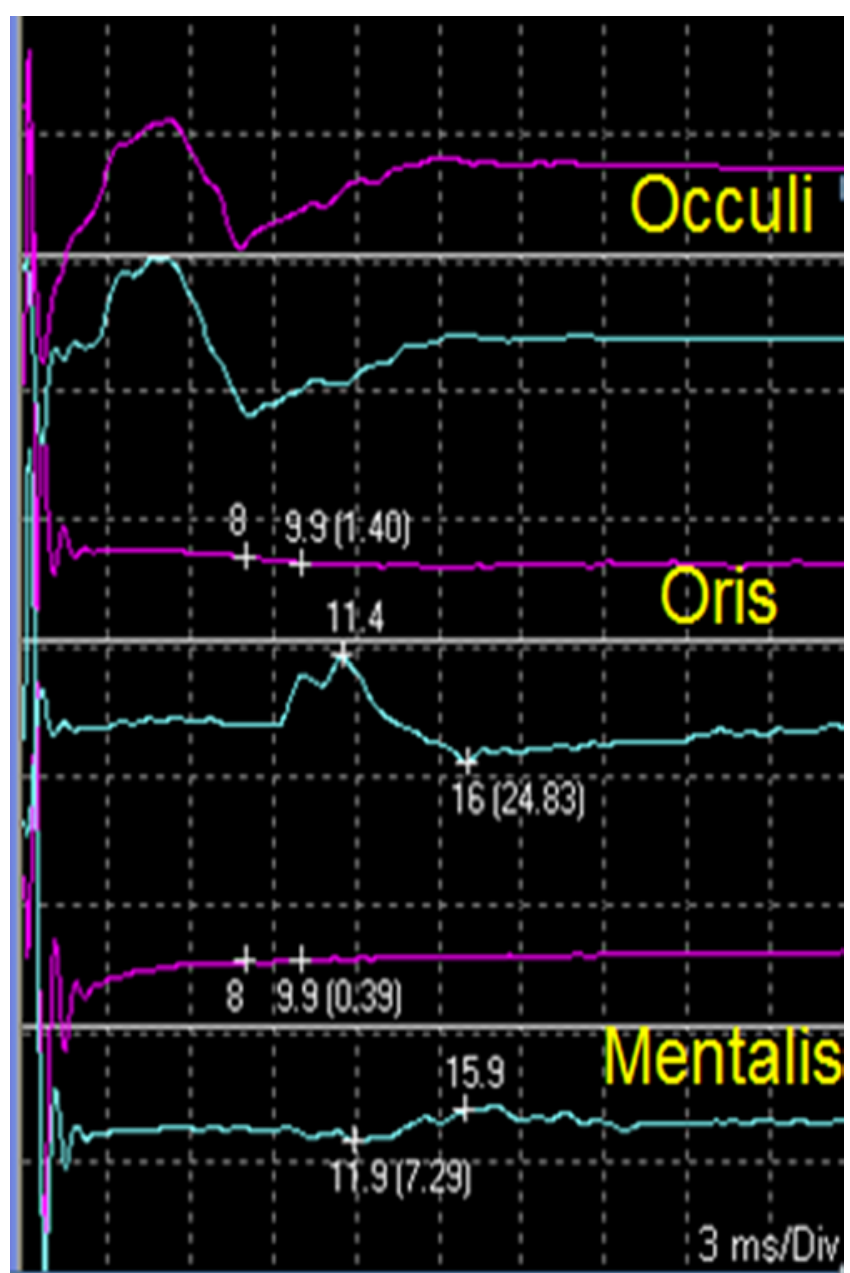

FIG. 2. Resolution of lateral spread seen with IONM. Aqua indicates the baseline, and magenta indicates the most recent trace. Figure is available in color online only.

data here are consistent with those findings, as the success rate was $93.8 \%$ when the LSR was abolished but only $70 \%$ when the LSR was not abolished. We report that the LSR was abolished in $61.5 \%$ of cases, which is consistent with the report of patients with prior Botox treatment. ${ }^{40}$

The fully endoscopic approach is limited in that the scope itself is prone to blood soiling and requires irrigation or antifogging. The scope also lacks 3D information feedback. At present, there is a lack of microinstruments specifically designed for endoscopic use in the subarachnoid space. These factors, along with the general lower frequency of use, contribute to the surgeon requiring a longer learning period to become proficient in the fully endoscopic technique to avoid complications. Identification of the neurovascular conflict in HFS is also surgeon dependent. The present study is limited in its generalizability due to the small sample size and lack of control groups. The retrospective nature further limits this study, given that not every single vessel was explicitly identified based on operative reports and that not all IONM information was readily available.

A study investigating outcomes among microsurgery, 
endoscope-assisted microsurgery, and fully endoscopic surgery would further elucidate the safety and feasibility of fully endoscopic MVD and would also directly compare outcomes of various techniques and be of great value. The senior author previously published the results of his 2-year transition period from using the conventional microscope to endoscope-assisted to fully endoscopic MVDs; there was no statistically significant difference in outcomes for the endoscopic approach when compared with microscopic procedures. ${ }^{11}$ In this 2-year transition period, all 5 patients with HFS had complete resolution of their preoperative symptoms. It is important to note that this transition period occurred only shortly after the endoscope was introduced into the senior author's routine practice. The purpose and outcome measures of this current study were designed to investigate the safety and feasibility, rather than the superiority, of fully E-MVD for HFS.

The present study found complete and near-complete resolution of symptoms in $60.7 \%$ and $14.3 \%$ of the cases, respectively. Patients in $7.1 \%$ of the cases experienced a $50 \%$ reduction in symptoms, and $3.6 \%$ had minimal reduction. No relief of symptoms was seen in $14.3 \%$ cases. This is lower than the reported success of $96.9 \%$ by Cheng et al. seen in endoscope-assisted and fully endoscopic MVD for HFS, ${ }^{4}$ but is comparable to the reported $70 \%-$ 95\% success from the general MVD literature regarding HFS. ${ }^{12,21,30,41}$ Long-term success rates (within 10 years) as high as $83 \%-97 \%$ have been reported. ${ }^{5,14}$ Of note, this follow-up is longer than that of our current cohort; in the cited studies, the proportion of spasm resolution increased with longer follow-up.

Visualization of the neurovascular conflict was seen in $100 \%$ of the cases in the present study. The endoscope allowed for complete visualization of the surgical region of interest. Video 1 shows a case in which the neurovascular conflict was only safely visualized with the $30^{\circ}$ endoscope and not with the $0^{\circ}$ scope alone.

VIDEO 1. Operative video showing a fully endoscopic approach for MVD for HFS. The lower cranial nerves are identified. The $30^{\circ}$ endoscope was used to provide safe visualization of the deeper anatomical structures. The AICA is visualized and lifted from the nerve, and Teflon is easily placed between the root entry zone and the AICA. Copyright University of Pennsylvania. Published with permission. Click here to view.

In our experience, the angled $30^{\circ}$ endoscope allows access to deeper structures that both the $0^{\circ}$ endoscope and the microscope are unable to reach safely without significant brainstem retraction. We hypothesize that the smaller durotomy required for the fully endoscopic technique may decrease postoperative headaches, which causes significant morbidity in this patient population. Future studies are needed to randomize patients into EA-MVD and EMVD to determine if clinically significant benefit, such as decreased postoperative headaches, can be achieved from a fully endoscopic technique.

\section{Conclusions}

The present study reports our experience with fully endoscopic MVD for HFS treatment. The overall purpose of this paper is to add to the existing data on fully endoscopic MVD. The endoscope provides excellent visualization and comprehensive evaluation of the neurovascular conflict in HFS patients. Exclusive use of the endoscope in MVD is a safe and feasible method of treating HFS. In the future, larger studies comparing arms of E-MVD, EA-MVD, and microscopic MVD will be of great value.

\section{Acknowledgments}

We thank the Neurosurgery Clinical Research Division, who contributed to the data collection and overall organization of this project. We would also like to thank Eo Trueblood for his original artwork contribution.

\section{References}

1. Abdeen K, Kato Y, Kiya N, Yoshida K, Kanno T: Neuroendoscopy in microvascular decompression for trigeminal neuralgia and hemifacial spasm: technical note. Neurol Res 22:522-526, 2000

2. Batla A, Goyal C, Shukla G, Goyal V, Srivastava A, Behari M: Hemifacial spasm: clinical characteristics of 321 Indian patients. J Neurol 259:1561-1565, 2012

3. Bohman LE, Pierce J, Stephen JH, Sandhu S, Lee JY: Fully endoscopic microvascular decompression for trigeminal neuralgia: technique review and early outcomes. Neurosurg Focus 37(4):E18, 2014

4. Cheng WY, Chao SC, Shen CC: Endoscopic microvascular decompression of the hemifacial spasm. Surg Neurol 70 (Suppl 1):S40-S46, 2008

5. Dannenbaum M, Lega BC, Suki D, Harper RL, Yoshor D: Microvascular decompression for hemifacial spasm: longterm results from 114 operations performed without neurophysiological monitoring. J Neurosurg 109:410-415, 2008

6. El Damaty A, Rosenstengel C, Matthes M, Baldauf J, Schroeder HW: The value of lateral spread response monitoring in predicting the clinical outcome after microvascular decompression in hemifacial spasm: a prospective study on 100 patients. Neurosurg Rev 39:455-466, 2016

7. El-Garem HF, Badr-El-Dine M, Talaat AM, Magnan J: Endoscopy as a tool in minimally invasive trigeminal neuralgia surgery. Otol Neurotol 23:132-135, 2002

8. Gardner WJ: Concerning the mechanism of trigeminal neuralgia and hemifacial spasm. J Neurosurg 19:947-958, 1962

9. Grundy BL, Jannetta PJ, Procopio PT, Lina A, Boston JR, Doyle E: Intraoperative monitoring of brain-stem auditory evoked potentials. J Neurosurg 57:674-681, 1982

10. Haines SJ, Torres F: Intraoperative monitoring of the facial nerve during decompressive surgery for hemifacial spasm. J Neurosurg 74:254-257, 1991

11. Halpern CH, Lang SS, Lee JY: Fully endoscopic microvascular decompression: our early experience. Minim Invasive Surg 2013:739432, 2013

12. Hanakita J, Kondo A: Serious complications of microvascular decompression operations for trigeminal neuralgia and hemifacial spasm. Neurosurgery 22:348-352, 1988

13. Ishikawa M, Ohira T, Namiki J, Ajimi Y, Takase M, Toya S: Abnormal muscle response (lateral spread) and F-wave in patients with hemifacial spasm. J Neurol Sci 137:109-116, 1996

14. Jannetta PJ, Abbasy M, Maroon JC, Ramos FM, Albin MS: Etiology and definitive microsurgical treatment of hemifacial spasm. Operative techniques and results in 47 patients. $\mathbf{J}$ Neurosurg 47:321-328, 1977

15. Jarrahy R, Berci G, Shahinian HK: Endoscope-assisted microvascular decompression of the trigeminal nerve. Otolaryngol Head Neck Surg 123:218-223, 2000

16. Jarrahy R, Cha ST, Eby JB, Berci G, Shahinian HK: Fully endoscopic vascular decompression of the glossopharyngeal nerve. J Craniofac Surg 13:90-95, 2002 
17. Jarrahy R, Eby JB, Cha ST, Shahinian HK: Fully endoscopic vascular decompression of the trigeminal nerve. Minim Invasive Neurosurg 45:32-35, 2002

18. Joo BE, Park SK, Cho KR, Kong DS, Seo DW, Park K: Realtime intraoperative monitoring of brainstem auditory evoked potentials during microvascular decompression for hemifacial spasm. J Neurosurg 125:1061-1067, 2016

19. Kang MC, Choi YS, Choi HK, Lee SH, Ghang CG, Kim $\mathrm{CH}$ : Efficacy of the disappearance of lateral spread response before and after microvascular decompression for predicting the long-term results of hemifacial spasm over two years. J Korean Neurosurg Soc 52:372-376, 2012

20. King WA, Wackym PA, Sen C, Meyer GA, Shiau J, Deutsch $\mathrm{H}$ : Adjunctive use of endoscopy during posterior fossa surgery to treat cranial neuropathies. Neurosurgery 49:108116, 2001

21. Kureshi SA, Wilkins RH: Posterior fossa reexploration for persistent or recurrent trigeminal neuralgia or hemifacial spasm: surgical findings and therapeutic implications. Neurosurgery 43:1111-1117, 1998

22. Lang SS, Chen HI, Lee JY: Endoscopic microvascular decompression: a stepwise operative technique. ORL J Otorhinolaryngol Relat Spec 74:293-298, 2012

23. Lee JYK, Pierce JT, Sandhu SK, Petrov D, Yang AI: Endoscopic versus microscopic microvascular decompression for trigeminal neuralgia: equivalent pain outcomes with possibly decreased postoperative headache after endoscopic surgery. J Neurosurg 126:1676-1684, 2017

24. Lee SH, Park BJ, Shin HS, Park CK, Rhee BA, Lim YJ: Prognostic ability of intraoperative electromyographic monitoring during microvascular decompression for hemifacial spasm to predict lateral spread response outcome. J Neurosurg 126:391-396, 2017

25. Lee SH, Song DG, Kim S, Lee JH, Kang DG: Results of auditory brainstem response monitoring of microvascular decompression: a prospective study of 22 patients with hemifacial spasm. Laryngoscope 119:1887-1892, 2009

26. Lu AY, Yeung JT, Gerrard JL, Michaelides EM, Sekula RF Jr, Bulsara KR: Hemifacial spasm and neurovascular compression. Sci World J 2014:349319, 2014

27. Magnan J, Chays A, Lepetre C, Pencroffi E, Locatelli P: Surgical perspectives of endoscopy of the cerebellopontine angle. Am J Otol 15:366-370, 1994

28. Miyazaki H, Deveze A, Magnan J: Neuro-otologic surgery through minimally invasive retrosigmoid approach: endoscope assisted microvascular decompression, vestibular neurotomy, and tumor removal. Laryngoscope 115:1612-1617, 2005

29. Montava M, Rossi V, CurtoFais CL, Mancini J, Lavieille JP: Long-term surgical results in microvascular decompression for hemifacial spasm: efficacy, morbidity and quality of life. Acta Otorhinolaryngol Ital 36:220-227, 2016

30. Nielsen VK: Pathophysiology of hemifacial spasm: I. Ephaptic transmission and ectopic excitation. Neurology 34:418426, 1984

31. Park JS, Kong DS, Lee JA, Park K: Hemifacial spasm: neurovascular compressive patterns and surgical significance. Acta Neurochir (Wien) 150:235-241, 2008

32. Polo G, Fischer C, Sindou MP, Marneffe V: Brainstem auditory evoked potential monitoring during microvascular decompression for hemifacial spasm: intraoperative brainstem auditory evoked potential changes and warning values to prevent hearing loss - prospective study in a consecutive series of 84 patients. Neurosurgery 54:97-106, 2004

33. Rak R, Sekhar LN, Stimac D, Hechl P: Endoscope-assisted microsurgery for microvascular compression syndromes. Neurosurgery 54:876-883, 2004

34. Sekula RF Jr, Bhatia S, Frederickson AM, Jannetta PJ, Quigley MR, Small GA, et al: Utility of intraoperative electro- myography in microvascular decompression for hemifacial spasm: a meta-analysis. Neurosurg Focus 27(4):E10, 2009

35. Tang CT, Kurozumi K, Pillai P, Filipce V, Chiocca EA, Ammirati M: Quantitative analysis of surgical exposure and maneuverability associated with the endoscope and the microscope in the retrosigmoid and various posterior petrosectomy approaches to the petroclival region using computer tomograpy-based frameless stereotaxy. A cadaveric study. Clin Neurol Neurosurg 115:1058-1062, 2013

36. Thirumala PD, Carnovale G, Habeych ME, Crammond DJ, Balzer JR: Diagnostic accuracy of brainstem auditory evoked potentials during microvascular decompression. Neurology 83:1747-1752, 2014

37. Thirumala PD, Shah AC, Nikonow TN, Habeych ME, Balzer JR, Crammond DJ, et al: Microvascular decompression for hemifacial spasm: evaluating outcome prognosticators including the value of intraoperative lateral spread response monitoring and clinical characteristics in 293 patients. J Clin Neurophysiol 28:56-66, 2011

38. von Eckardstein K, Harper C, Castner M, Link M: The significance of intraoperative electromyographic "lateral spread" in predicting outcome of microvascular decompression for hemifacial spasm. J Neurol Surg B Skull Base 75:198-203, 2014

39. Wang A, Jankovic J: Hemifacial spasm: clinical findings and treatment. Muscle Nerve 21:1740-1747, 1998

40. Wang X, Thirumala PD, Shah A, Gardner P, Habeych M, Crammond DJ, et al: Effect of previous botulinum neurotoxin treatment on microvascular decompression for hemifacial spasm. Neurosurg Focus 34(3):E3, 2013

41. Wilkins RH: Hemifacial spasm: a review. Surg Neurol 36:251-277, 1991

42. Yamashita S, Kawaguchi T, Fukuda M, Suzuki K, Watanabe M, Tanaka R, et al: Lateral spread response elicited by double stimulation in patients with hemifacial spasm. Muscle Nerve 25:845-849, 2002

43. Ying T, Thirumala P, Gardner P, Habeych M, Crammond D, Balzer J: The incidence of early postoperative conductive hearing loss after microvascular decompression of hemifacial spasm. J Neurol Surg B Skull Base 76:411-415, 2015

\section{Disclosures}

The authors report no conflict of interest concerning the materials or methods used in this study or the findings specified in this paper.

\section{Author Contributions}

Conception and design: Flanders, Petrov, Lee. Acquisition of data: Flanders, McShane, Tambi, Lee. Analysis and interpretation of data: Flanders, Tambi, Lee. Drafting the article: Flanders, Wilent. Critically revising the article: Flanders, Blue, Wilent, Lee. Reviewed submitted version of manuscript: all authors. Approved the final version of the manuscript on behalf of all authors: Flanders. Statistical analysis: Flanders, Wilent, Tambi, Lee. Administrative/technical/material support: Roberts, McShane, Wilent, Tambi, Petrov, Lee. Study supervision: Lee. Submission of IRB: McShane.

\section{Supplemental Information Videos}

Video 1. https://vimeo.com/274685949.

\section{Correspondence}

Tracy M. Flanders: University of Pennsylvania, Philadelphia, PA. tracy.flanders@uphs.upenn.edu. 